\begin{tabular}{lllll}
\multicolumn{5}{l}{ Abstract 183 Table 1} \\
\hline & $\%$ Asymmetry HT & $\%$ Asymmetry ST & $\%$ Asymmetry CT & $5 \mathrm{~m}(\mathrm{sec})$ \\
\hline After warm up & $10.2 \pm 10.1$ & $5.2 \pm 3.1$ & $5.1 \pm 5.5$ & $1.31 \pm 0.05$ \\
After series 1 & $11.3 \pm 9.6$ & $17.1 \pm 17.6^{* *}$ & $13.1 \pm 5.5^{* *}$ & $1.52 \pm 0.11^{*}$ \\
After series 2 & $14.1 \pm 7.7$ & $20.2 \pm 16.7^{* *}$ & $15.1 \pm 9.8^{* *}$ & $1.59 \pm 0.07^{*}$ \\
After series 3 & $14.7 \pm 7.6$ & $19.9 \pm 15.1^{* *}$ & $20.2 \pm 5.6^{* *}$ & $1.72 \pm 0.13^{*}$ \\
\hline${ }^{*} \mathrm{p}<0.05^{* *} \mathrm{p}<0.01 \mathrm{p}<0.001$ (from after-warm-up value)
\end{tabular}

Conclusions The jumps with frontal plane displacement and those on the two planes (the CT is perfomed by jumping simultaneously on the sagittal and frontal plane) have the highest asymmetry values and seem influenced by post-SSG fatigue. Sprint values undergo acute decreases in performance too.

\section{MANAGING VITAMIN D SUPPLEMENTATION IN ELITE ACADEMY FOOTBALLERS}

Amit Verma, Thomas Maynard, Jim Moxon. Liverpool Football Club, Liverpool, UK

\subsection{6/bjsports-2021-IOC. 169}

Background The maintenance of phosphate and calcium homeostasis alongside optimal vitamin D status is essential for bone health. Moreover, low vitamin D is associated with an increased risk of stress fracture, impaired muscular remodelling and increased risk of upper respiratory tract infections.

Objective The primary objective was to assess the efficacy of an individualised in-season vitamin D supplementation programme. The secondary aim was to observe for regression of vitamin D levels during the longer daylight months (AprilOctober) in players where vitamin D was not routinely supplemented.

Design Serum 25 Hydroxyvitamin D (Serum 25[OH]D) levels were collected twice a year (October and April). Levels were categorised as: deficient $(<30 \mathrm{nmol} / \mathrm{l})$, insufficient (31-75), sufficient (76-175) or overloaded (>175). Players with sufficient levels were given a maintenance protocol $(20,000 \mathrm{iu}$ once weekly) October-April. Players who were deficient/insufficient were given a loading regimen (20,000iu twice weekly for three weeks) and the maintenance protocol thereafter.

Setting A category one Premier League Elite Player Performance Plan (EPPP) youth football academy.

Participants 13 participants from the $\mathrm{u} 23 \mathrm{~s}$ and $\mathrm{u} 18 \mathrm{~s}$ squads.

Interventions Serum 25[OH]D levels were collected (via venous blood sample) twice a year (October and April). Levels were categorised as: deficient $<30 \mathrm{nmol} / 1$, insufficient $31-75 \mathrm{nmol} / \mathrm{l}$, sufficient $76-175 \mathrm{nmol} / 1$ or overloaded $>175 \mathrm{nmol} / \mathrm{l}$.

Players with sufficient or insufficient levels were given a maintenance protocol (20,000IU once weekly) during OctoberApril. Players who were deficient were given a loading regimen (20,000iu twice weekly for three weeks) and the maintenance protocol for the remainder of winter ( 22 weeks). Oral supplementation was administered using Synergy Biologics LTD Pro D3 Sport 20k (UK).

Results There was no significant increase in serum $25[\mathrm{OH}] \mathrm{D}$ during the supplementation period (October-April). However, 20,000iu of supplemental Vitamin D over a 25-week period was sufficient to maintain vitamin D status. Baseline serum 25[OH]D was not significantly increased after a period of supplemental vitamin D during winter months. Further studies in this population group would be beneficial to assess the effects of longer term season-on-season supplementation.

\section{REVIEW OF THE PHYSIOTHERAPY SERVICE FOR ATHLETES IN 2018 OLYMPIC WINTER GAMES}

${ }^{1} J$ oon Young Chang, ${ }^{1,2}$ Young Hee Lee, ${ }^{3,4}$ Marie-Elaine Grant, ${ }^{7}$ Jong Ha Lee, ${ }^{5}$ Joshua Sung H You, ${ }^{6}$ Tae Gyu Kim, ${ }^{1,8}$ In Deok Kong. ${ }^{1}$ Yonsei Institute of Sports Science and Exercise Medicine, Wonju, South Korea (Republic of); ${ }^{2}$ Rehabilitation Medicine, Wonju College of Medicine, Yonsei University, Wonju, South Korea (Republic of); ${ }^{3}$ International Olympic Committee, Lusanne, Switzerland; 'Institute of Sport and Health, University College Dublin, Dublin, Ireland; ${ }^{5}$ Sports Movement Artificial-Intelligence Robotics Technology (SMART) Institute, Department of Physical Therapy, Yonsei University, Wonju, South Korea (Republic of); ${ }^{6}$ Department of Marine Sports, Pukyong National University, Busan, South Korea (Republic of); ${ }^{7}$ Department of Rehabilitation Medicine, Kyung Hee University College of Medicine and Hospital, Seoul, South Korea (Republic of); ${ }^{8}$ Department of Physiology, Yonsei University Wonju College of Medicine, Wonju, South Korea (Republic of)

\subsection{6/bjsports-2021-IOC.170}

Objective This study will provide to better understand the needs for physiotherapy services during the 2018 PyeongChang Olympic Winter Games (POG) from two polyclinics. It is necessary to understand the needs and what physiotherapists do during the Olympic Winter games for first time.

Design An observational study.

Setting 2018 PyeongChang Olympic Winter Games.

Participants Athletes who visited the physiotherapy department of polyclinics.

Results During 25 the days of the POG, a total of 125 athletes ( $\mathrm{n}=125,83$ males, 42 females) visited the two polyclinics. Of all visits, $69.6 \%$ were from the mountain polyclinic and $30.4 \%$ from the city. There were three reasons for visit, most of the reason for visit was injury and injury with recovery or injury prevention. Overall, the injury rate (per 1000 athletes) was 42.8 across 13 sports visited the physiotherapy department during the POG. Total numbers of treatments sessions were 823 provided and electrophysical modalities $(36.2 \%)$ was the most utilized service in POG. And also there were significant differences in the physiotherapy services provided at the two polyclinics.

Conclusion As each polyclinic differed in location, they addressed different populations of athletes; hence, the study provides insights into the injury trends and different physiotherapy treatments.

\section{EPIDEMIOLOGY OF INJURY AND RETIREMENT FROM SPORT AMONG FORMER INTERNATIONAL ATHLETES}

${ }^{1}$ Dale Cooper, ${ }^{2}$ Mark Batt, ${ }^{3}$ Debbie Palmer. ' School of Allied Health Professions, University of Leicester, Leicester, UK; ${ }^{2}$ Centre for Sport, Exercise and Osteoarthritis Research Versus Arthritis, Queen's Medical Centre, Nottingham, UK; ${ }^{3}$ Institute of Sport, PE and Health Sciences, Moray House School of Education and Sport, University of Edinburgh, Edinburgh, UK

\subsection{6/bjsports-2021-IOC.171}

Background High-level athletes are at an increased injury risk. Yet relatively little is known about the prevalence of careerending injuries in high-level sport. 
Objective To determine in retired international athletes: (1) the prevalence of significant injury and early retirement, and (2) if those with generalised joint hypermobility $(\mathrm{GJH})$ are more likely to sustain a significant injury.

Design Cross-sectional study.

Setting Data from a survey of retired Olympic athletes.

Patients (or Participants) 605 retired athletes, aged 40-97 years, $40 \%$ female $(n=244)$ who had competed internationally for Great Britain at the Summer and/or Winter Olympic Games.

Interventions (or Assessment of Risk Factors) Data collected on reasons for retirement from competitive sport including the type and location of significant injury. A validated line drawing instrument was used to measure generalised joint hypermobility (GJH) (Beighton > 3/9).

Main Outcome Measurements Injury-forced retirement from a competitive sports career.

Results Of those who replied to the questionnaire $(\mathrm{n}=714)$, $84.7 \%(\mathrm{n}=605)$ were retired from sport, and 21.8\% ( $\mathrm{n}=$ 132) of those retired from sport reported that they had retired early because of injury. The main locations of injuries that were reported to be responsible for retirement from sport were the lower back (25.8\%), knee (25.0\%), lower leg (8.3\%), ankle (7.6\%), Achilles tendon (6.8\%), shoulder $(6.8 \%)$, hip $(5.3 \%)$, and thigh $(5.3 \%)$. The main types of injury reported to be responsible for early retirement from sport were soft tissue related (23.9\%), injuries to the intervertebral disc (19.0\%), intervertebral joint (18.2\%), and cartilage injuries at the hip and knee (9.1\%). Overall, GJH was not associated with a significant injury in female [OR 1.25; 95\% CI, 0.62-2.50] or male athletes [OR 0.76; 95\% CI, 0.35$1.65]$.

Conclusions Injury is a major cause of early retirement among high-level athletes. However, those with GJH were no more likely to sustain a significant injury.

\section{EPIDEMIOLOGY AND IMPACT OF TRAVELLER'S DIARRHOEA ON PARTICIPANTS OF THE PRE-OLYMPIC TEST EVENT 'READY STEADY TOKYO 2019'}

${ }^{1}$ Nebojša Nikolić, ${ }^{2}$ Sergio Migliorini, ${ }^{3}$ Lidija Bilić-Zule. 'World Sailing Medical Commission, London, UK; ${ }^{2}$ International Triathlon Union Medical Commission, Lausanne, Switzerland; ${ }^{3}$ University of Rijeka, Faculty of Medicine, Rijeka, Croatia

\subsection{6/bjsports-2021-IOC.172}

Background Mass gatherings at major sport events such as Olympic Games pose unique health risks because having the large number of people in a small space can aid the spread of infectious diseases. That can also pose risks for athletes' health and can endanger their performance. Water quality testing of the fields of play (FOP) in Tokyo are showing potential environmental problems (temperature and water pollution) that could endanger the health and safety of the athletes. It is necessary to develop adequate preventive and mitigation measures that would be used in Tokyo Olympics. This research aims to provide international and local authorities with evidence-base for such an intervention.

Hypothesis Athletes of open water sports are more prone to gastrointestinal infections (TD) due to the exposure of polluted water on the FOP. This hypothesis has been tested by investigating data obtained from the survey and identifying the influence of contact with the water of the FOP on athletes' health.

Design Cross sectional study.

Setting Pre-Olympic test event Ready Steady Tokyo 2019 (1722 August)

Patients (or Participants) athletes (sailing and triathlon) and their accompanying teams on shore.

Main Outcome Measurements Surveys among athletes in contact with the water and their accompanying teams on shore. The whole population defined in investigation was approached. Anonymous TD questionnaire was distributed in hard copy and collected on the last day of the event. The online version was available for one month after the event for participants that omitted to fulfill it on site. Data were processed using MedCalc statistical software. The incidence of diarrhea, its duration, the number of stools, impact on performance was recorded.

Results The study presents the epidemiology and the impact of TD to athletes participating at Pre-Olympic test event Ready Steady Tokyo 2019.

Conclusions Conclusions on risk are made and compared with the data from Rio 2016 Olympics.

\section{THE RETIRED OLYMPIAN MUSCULOSKELETAL HEALTH STUDY (ROMHS) COHORT: RECRUITMENT OF 3,357 OLYMPIANS AND 1,735 GENERAL POPULATION CONTROLS}

${ }^{1,2}$ Debbie Palmer, ${ }^{3}$ Dale Cooper, ${ }^{4}$ Carolyn Emery, ${ }^{5,6}$ Mark Batt, 7,8 Lars Engebretsen, ${ }^{6,9}$ Brigitte Scammell, ${ }^{8}$ Torbjørn Soligard, ${ }^{7}$ Kathrin Steffen, ${ }^{4,10}$ Jackie Whittaker, ${ }^{8}$ Richard Budgett. 'School of Applied Sciences, Edinburgh Napier University, Edinburgh, UKi ${ }^{2}$ Institute for Sport, PE and Health Sciences, Moray House School of Education and Sport, University of Edinburgh, Edinburgh, UK; ${ }^{3}$ School of Allied Health Professions, University of Leicester, Leicester, UK; ${ }^{4}$ Sport Injury Prevention Research Centre, Faculty of Kinesiology, University of Calgary, Calgary, Canada; ${ }^{5}$ Nottingham University Hospitals Trust, Nottingham, UK; ${ }^{6}$ Centre for Sport, Exercise and Osteoarthritis Versus Arthritis, Queens Medical Centre, Nottingham, UK; ${ }^{7}$ Department of Sports Medicine, Oslo Sports Trauma Research Centre, Norwegian School of Sport Sciences, Oslo, Norway; ${ }^{8}$ Medical and Scientific Department, International Olympic Committee, Lausanne, Switzerland; ${ }^{9}$ School of Medicine, University of Nottingham, Nottingham, UK; ${ }^{10}$ Department of Physical Therapy, University of British Columbia, Vancouver, Canada

\subsection{6/bjsports-2021-IOC.173}

Background Elite athletes are exposed to high physiological training and competition loads and associated increased injury risk. While there is a wealth of evidence reporting the prevalence of injuries seasonally and during major games events, there are few studies examining injury risk across an elite athlete's entire sporting-career.

Objective Recruit a representative sample of Olympians who have retired from elite competition, and general population controls, in order to examine Olympic-career injury patterns and risk, and their association with current health status.

Design Cross-sectional survey of sporting and injury history, and general health.

Setting Olympians and the general population, from 209 countries worldwide.

Patients (or Participants) Athletes who had competed at a Summer and/or Winter Olympic Games and considered themselves retired from Olympic level training and competition; 